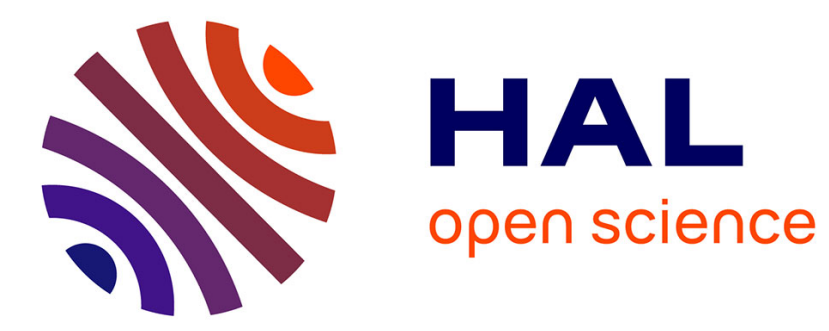

\title{
Shortest paths for the Dubins' vehicle in heterogeneous environments
}

\author{
Bruno Hérissé, Romain Pepy
}

\section{To cite this version:}

Bruno Hérissé, Romain Pepy. Shortest paths for the Dubins' vehicle in heterogeneous environments. 52nd IEEE Conference on Decision and Control, Dec 2013, Florence, Italy. pp.4504-4509. hal00861238v2

\section{HAL Id: hal-00861238 \\ https://hal.science/hal-00861238v2}

Submitted on 25 Jul 2014

HAL is a multi-disciplinary open access archive for the deposit and dissemination of scientific research documents, whether they are published or not. The documents may come from teaching and research institutions in France or abroad, or from public or private research centers.
L'archive ouverte pluridisciplinaire HAL, est destinée au dépôt et à la diffusion de documents scientifiques de niveau recherche, publiés ou non, émanant des établissements d'enseignement et de recherche français ou étrangers, des laboratoires publics ou privés. 


\title{
Shortest paths for the Dubins' vehicle in heterogeneous environments
}

\author{
Bruno Hérissé, Romain Pepy
}

\begin{abstract}
In this paper, the problem of finding minimum length paths for a Dubins' vehicle that can only move forward in an heterogeneous environment is considered. An hybrid version of the Pontryagin's maximum principle is used to derive necessary conditions for optimality. Unlike in the case of homogeneous environments, it is deduced that heterogeneity of the environment implies that optimal paths can contain reflections. A subclass of environments is analyzed more specifically in order to obtain additional necessary conditions. Based on these results, two concrete application cases are detailed to demonstrate the usefulness of the approach in practice. The first example concerns a mobile robot and the second example concerns a glider.
\end{abstract}

\section{INTRODUCTION}

The purpose of this paper is to characterize shortest paths for a vehicle moving only forward in an heterogeneous 2dimensional Cartesian plane. The environment is heterogeneous in the sense that the minimum turning radius that the robot can perform depends on the vehicle position along one axis of the plane. Thus, the vehicle dynamics are governed by (see Fig. 1)

$$
\left\{\begin{array}{l}
\dot{x}=v \cos \theta \\
\dot{z}=v \sin \theta \\
\dot{\theta}=v c(z) u, \quad|u| \leq 1,
\end{array}\right.
$$

where $(x, z) \in \mathbb{R}^{2}$ is the vehicle position, $\theta \in \mathbb{R}$ is the angle between the vehicle and the $\mathrm{x}$-axis, $v>0$ is the vehicle velocity, $u \in \mathbb{R}$ is the control input ( $u \in[-1,1]$ ) and $c(z) \in \mathbb{R}_{+}$is the maximum curvature the robot can perform at vertical position $z$. The dynamics of the forward velocity $v$ is not specified, the analysis in this paper only considers the shortest path problem.

When the maximum curvature $c$ is constant, i.e. the environment is homogeneous, the vehicle verifying (1) is known as the Dubins' car and finding the shortest path between two configurations $(x, z, \theta)$ is known as the Dubins' problem [4]. This problem was solved in [4] using only geometric arguments. It states that shortest paths are either of type $C C C$ or $C S C$, that is a concatenation of three arcs of a circle with radius $1 / c$ or a concatenation of an arc of a circle with radius $1 / c$, a line segment and another arc of a circle with radius $1 / c$. Later, Dubins' results were proved using Pontryagin's maximum principle [1]. The 3D extension of Dubins' paths was considered in [11].

Dubins' work gave rise to many other similar problems. For example, shortest paths for a robot moving forward

B. Hérissé and R. Pepy are with Onera - The French Aerospace Lab, F-91761 Palaiseau, France (email: bruno.herisse@onera.fr, romain.pepy@onera.fr) and backward were derived [9]. In [1], [13], similar results were recovered using Pontryagin's maximum principle [8]. In [2], the derivative of the curvature is controlled and assumed to be bounded. In [5], [6], the effect of a constant wind is analyzed for unmanned aerial vehicles with the same dynamics as the Dubins' car. In [3], an anisotropic environment is considered, that is the maximum curvature $c$ depends on the orientation $\theta$ of the vehicle.

As far as the authors know, only one previous work addresses the optimal problem for a Dubins' vehicle in an heterogeneous environment. In [10], the environment consists of two half-planes where the robot has two different velocities and where it can maneuver with the same turning rate. A generalized refraction law is derived from the characterization of minimum time paths. The problem presented here is different from [10] since it addresses the shortest path problem. Moreover, in [10], the vehicle moves in a terrain with heterogeneous velocity while in the present paper, this is the maneuverability of the vehicle that is heterogeneous along the terrain. In addition, the environment is not restricted to only two media in the present paper, i.e. the maximum curvature $c(z)$ can be discontinuous for an unlimited number of positions $z$.

In this paper, since discontinuous environments are also considered, the classical Pontryagin's maximum principle cannot be used. A more general version of the maximum principle [12], suitable for hybrid control problems, is used to derive necessary conditions for optimality. From these conditions, some features of optimal paths are deduced. Shortest paths are shown to be a concatenation of line segments $S$ and arcs $C$ of maximum curvature. Moreover, it is shown that shortest paths can contain reflections, i.e. paths containing two different line segments with opposite orientation angles are candidates for optimality. The case where the maximum curvature $c(z)$ is a monotonic function of $z$ is analyzed. In that case, it is proved that optimal paths containing line segments are either of type $C S C$ or $C S C S C$ if both line segments have opposite orientation angles.

Based on these results, two application cases are presented. First, a mobile robot is considered. The terrain consists of two half-planes with two different coefficients of friction implying two maximum steering angles to ensure rolling without slipping. Second, a glider moving in the vertical plane is considered. Due to the exponential decreasing of atmospheric pressure with altitude, the maximum lift force is also exponentially decreasing with altitude.

The body of the paper consists of four sections followed by a conclusion. Section II presents the problem and notations. 


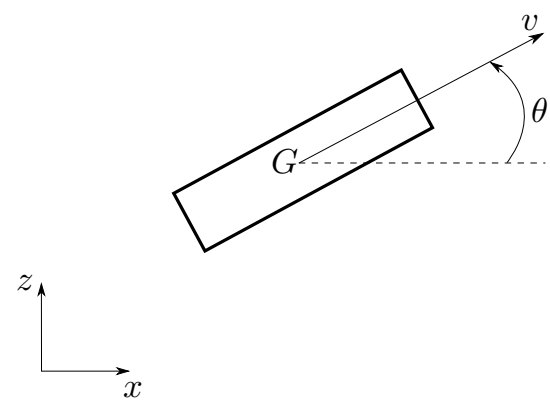

Fig. 1. Vehicle model.

In Section III, conditions for optimality are enumerated and the case where the maximum curvature $c(z)$ is a monotonic function of $z$ is detailed. Sections IV and V present the two examples of application for the problem addressed in this paper.

\section{Problem Statement}

In this paper, a Dubins' vehicle moving forward with bounded curvature across a $2 \mathrm{D}$ heterogeneous environment is considered. A coordinate system $(x, z, \theta) \in \mathbb{R}^{3}$ is used to determine the position $(x, z)$ of the center of mass $G$ and the orientation $\theta$ of the vehicle in this environment (see Fig. 1). The robot dynamics are given by (1). The maximum curvature $c(z)$ in (1) is assumed to satisfy the two following conditions:

Assumption 2.1: $c(z)$ is discontinuous for a finite number $N \in \mathbb{N}$ of positions $z_{q}, q \in \mathcal{Q}=\llbracket 1, N \rrbracket$.

Assumption 2.2: If $N \neq 0, c(z)$ is $C^{1}$ in each of the intervals $\left(-\infty, z_{1}\right],\left[z_{q}, z_{q+1}\right], q \in \llbracket 1, N-1 \rrbracket$ and $\left[z_{N},+\infty\right)$. It is $C^{1}$ in $\mathbb{R}$ otherwise.

The problem addressed in the paper is to characterize trajectories of minimum length for the system (1) given initial and final states $\left(x_{0}, z_{0}, \theta_{0}\right)$ and $\left(x_{f}, z_{f}, \theta_{f}\right)$, that is trajectories minimizing the functional

$$
s_{f}=\int_{0}^{t_{f}} v d t
$$

where $t_{f}$ is the final time assumed to be free (free interval optimal control problem) and $s_{f}$ is the length of the path.

Since we are interested in minimum length trajectories (the dynamics of the forward velocity $v$ is not specified), it is convenient to make a change of variable from time $t$ to curvilinear abscissa $s(t)=\int_{0}^{t} v(u) d u$. Thus, dynamics can be re-written as follows:

$$
\left\{\begin{array}{l}
x^{\prime}=\frac{d x}{d s}=\cos \theta \\
z^{\prime}=\frac{d z}{d s}=\sin \theta \\
\theta^{\prime}=\frac{d \theta}{d s}=c(z) u, \quad|u| \leq 1
\end{array}\right.
$$

Therefore, the problem consists in minimizing $s_{f}$, the path length. Notice that in the case when the forward velocity $v$ is constant, minimizing the path length $s_{f}$ is equivalent to minimizing the final time $t_{f}$.

\section{OPTIMALITY CONDITIONS}

\section{A. The general case}

The problem stated in Section II is similar to the classical Dubins' problem and can also be solved using classical optimal control theory [1] provided that the system (2) is sufficiently regular [8]. Assumption 2.1 prevents from using this version of the Pontryagin's maximum principle. In the followings, the more general version of the maximum principle described in [12] is used. Notice that this principle was first used in [10] to analyze an analogous problem.

Before enumerating necessary conditions for optimality, curvilinear abscissae where the system crosses a discontinuity along the path, i.e. curvilinear abscissae where $c(z)$ meets a discontinuity, have to be defined.

Definition 3.1: A switching curvilinear abscissa $S$ along a path is defined as a curvilinear abscissa verifying the following condition: there exists $q \in \mathcal{Q}$ such that $z(S)=z_{q}$ and there exists $\varepsilon>0$ such that $z(s \in[S-\varepsilon, S)) \neq z_{q}$. Applying the maximum principle presented in [12] to the optimal control problem described in Section II, the following result is obtained.

Proposition 3.2: Consider a solution $\boldsymbol{\xi}=(x, z, \theta)^{\top}$ to the shortest path problem for the system (2) with control input $u$ and minimum length $s_{f}$. Assume that the number of switching curvilinear abscissae is $J-1, J \in \mathbb{N}^{*}$. Then, there exists $\lambda_{0} \in \mathbb{R}$ and $J$ absolutely continuous functions $\lambda^{j}:\left[s_{j}, s_{j+1}\right] \rightarrow \mathbb{R}^{3}, j \in \llbracket 0, J-1 \rrbracket$ such that

1) $\lambda_{0}>0$ or $\lambda^{j} \neq 0, \forall j \in \llbracket 0, J-1 \rrbracket$;

2) $\forall j \in \llbracket 0, J-1 \rrbracket$,

$$
\left(\boldsymbol{\lambda}^{j}\right)^{\prime}=-\frac{\partial H^{j}}{\partial \boldsymbol{\xi}}, \quad \forall s \in\left[s_{j}, s_{j+1}\right]
$$

where

$$
H^{j}=\lambda_{0}+\lambda_{1}^{j} \cos \theta+\lambda_{2}^{j} \sin \theta+\lambda_{3}^{j} c(z) u
$$

and $\lambda^{j} \stackrel{\text { def }}{=}\left(\lambda_{1}^{j}, \lambda_{2}^{j}, \lambda_{3}^{j}\right)^{\top}$

3) $\forall j \in \llbracket 0, J-1 \rrbracket, \forall s \in\left[s_{j}, s_{j+1}\right], H^{j}(s)=0$;

4) If $J \geq 2, \forall j \in \llbracket 0, J-2 \rrbracket, \lambda_{1}^{j}\left(s_{j+1}\right)=\lambda_{1}^{j+1}\left(s_{j+1}\right)$ and $\lambda_{3}^{j}\left(s_{j+1}\right)=\lambda_{3}^{j+1}\left(s_{j+1}\right)$

5) $\forall(s, j), u=-\operatorname{sgn}\left(\lambda_{3}^{j}\right)$, where $\operatorname{sgn}(x)=x /|x|, \forall x \in$ $\mathbb{R}^{*}$ and $\operatorname{sgn}(0)=0$.

Notice that in Proposition 3.2, switching curvilinear abscissae are every $s_{j}, j \in \llbracket 1, J-1 \rrbracket(J \geq 2)$ and $s_{f}=s_{J}$. First item of Proposition 3.2 follows from non-triviality condition of the maximum principle. Item 2 is the adjoint equation. Item 3 is due to the Hamiltonian value condition and the fact that a free interval optimal control problem is addressed in this paper. Item 4 is a direct application of the switching condition described in [12]. As for Item 5, it follows from Hamiltonian minimization.

Corollary 3.3: From Proposition 3.2, the followings can be deduced:

- $\forall j \in \llbracket 0, J-1 \rrbracket, \lambda_{1}^{j}=\lambda_{1}$ is a constant. The proof is straightforward using Items 2 and 4: for $j \in \llbracket 0, J-1 \rrbracket$, $\left(\lambda_{1}^{j}\right)^{\prime}=0$ in $\left[s_{j}, s_{j+1}\right]$ and $\lambda_{1}^{j}\left(s_{j+1}\right)=\lambda_{1}^{j+1}\left(s_{j+1}\right)$ 
at switching curvilinear abscissae $s_{j+1}, j \in \llbracket 0, J-2 \rrbracket$ $(J \geq 2)$

- Item 5 implies that optimal paths are a concatenation of line segments $S$ (when $\lambda_{3}^{j} \equiv 0$ on a subinterval of $\left[s_{j}, s_{j+1}\right]$ ) and arcs of maximum curvature $C$ (when $\lambda_{3}^{J} \neq 0$ );

- If the optimal path contains a line segment with orientation $\theta=\alpha$, then there can exist another line segment with orientation $\theta=-\alpha$ (modulo $2 \pi$ ) along the path. This follows from the fact that $\lambda_{2}$ can vary along the path, i.e. $\lambda_{2}^{j}(j \in \llbracket 0, J-1 \rrbracket)$ can vary in $\left[s_{j}, s_{j+1}\right]$ and $\lambda_{2}^{j}\left(s_{j+1}\right)$ is not necessarily equal to $\lambda_{2}^{j+1}\left(s_{j+1}\right)$ at switching curvilinear abscissae $s_{j+1}, j \in \llbracket 0, J-2 \rrbracket$ $(J \geq 2)$. This type of optimal path containing two line segments with opposite orientation angles will be said to contain reflections;

- Items 3 and 4 imply that if $\lambda_{3}^{j}\left(s_{j+1}\right)=0$ and $\sin \left(\theta\left(s_{j+1}\right)\right) \neq 0, \quad j \in \llbracket 0, J-2 \rrbracket(J \geq 2)$, then $\lambda_{2}^{j}\left(s_{j+1}\right)=\lambda_{2}^{j+1}\left(s_{j+1}\right)$. This implies that the orientation of a line segment cannot change when the system crosses a discontinuity.

Proof: The third point of Corollary 3.3 is proved here. Assume that the optimal path $\boldsymbol{\xi}$ contains a line segment with orientation $\theta=\alpha$. Then, there exists $j \in \llbracket 0, J-1 \rrbracket$ such that $\lambda_{3}^{j} \equiv 0$ on a subinterval $\mathcal{I}$ of $\left[s_{j}, s_{j+1}\right]$. From Items 2 and 3 of Proposition 3.2, it is straightforward to show that $\lambda_{1}^{j}=-\lambda_{0} \cos (\alpha)$ and $\lambda_{2}^{j}=-\lambda_{0} \sin (\alpha)$ in $\mathcal{I}$. From Item 1 , it can be deduced that $\lambda_{0} \neq 0$. Moreover, since $\lambda_{1}^{j}=\lambda_{1}$ is a constant, if there exists another line segment along the path with orientation $\theta=\beta$ then $\cos (\beta)=\cos (\alpha)$, hence $\beta= \pm \alpha$ (modulo $2 \pi$ ).

Existence of reflections in optimal paths is the main result obtained here. There cannot exist such reflections for the classical Dubins' vehicle with a constant maximum curvature. In [10], only optimal paths with one switching curvilinear abscissa are described, therefore, reflections are not outlined.

The result stated below considers more specific systems characterized by the fact that $c(z)$ is a monotonic function of $z$. This will be used in the two examples presented in the next two sections.

\section{B. The monotonic case}

Theorem 3.4: Assume that $c(z)$ is a monotonic function of $z$. Then, any optimal paths $\xi$ containing a line segment can contain at most one other line segment with opposite orientation angle.

Theorem 3.4 means that an optimal path can contain one or two line segments and that an optimal path containing two line segments is necessarily a path with a unique reflection. Proof: Assume that $c(z)$ is a decreasing function of $z$. The result is similar for an increasing function.

First, we show that $\lambda_{2}$ is decreasing. To this purpose, switching curvilinear abscissae and intervals where the system is continuous are analyzed separately. The proof follows from Items 2 and 3 of Proposition 3.2.
- $\forall j \in \llbracket 0, J-1 \rrbracket, \forall s \in\left[s_{j}, s_{j+1}\right],\left(\lambda_{2}^{j}\right)^{\prime}=-\lambda_{3}^{j} \frac{d c}{d z} u$. Since $u=-\operatorname{sgn}\left(\lambda_{3}^{j}\right)$, this implies that

$$
\left(\lambda_{2}^{j}\right)^{\prime}=\left|\lambda_{3}^{j}\right| \frac{d c}{d z} \leq 0,
$$

Therefore, $\lambda_{2}^{j}$ is decreasing;

- $\forall j \in \llbracket 0, J-2 \rrbracket$, for every curvilinear abscissae $s=$ $s_{j+1}$,

$$
\begin{aligned}
& \lambda_{2}^{j} \sin \left(\theta^{j}\right)-\left|\lambda_{3}^{j}\right| c^{j}= \\
& \lambda_{2}^{j+1} \sin \left(\theta^{j+1}\right)-\left|\lambda_{3}^{j+1}\right| c^{j+1}
\end{aligned}
$$

where $\theta^{j}=\theta\left(s_{j}\right)$ and $c^{j}=c\left(s_{j}\right)$. Therefore, since $\lambda_{3}^{j}=\lambda_{3}^{j+1}$ and $\theta^{j}=\theta^{j+1}$,

$$
\left(\lambda_{2}^{j+1}-\lambda_{2}^{j}\right) \sin \left(\theta^{j}\right)=-\left|\lambda_{3}^{j}\right|\left(c^{j}-c^{j+1}\right)
$$

It is straightforward to see that if $\sin \theta^{j} \neq 0$, then $\sin \left(\theta^{j}\right)$ is of the same sign as $\left(c^{j}-c^{j+1}\right)$. It follows that $\lambda_{2}^{j+1} \leq \lambda_{2}^{j}$.

In addition, using Item 3 of Proposition 3.2, it follows that

$$
\frac{\lambda_{2}^{j+1}}{c^{j+1}}-\frac{\lambda_{2}^{j}}{c^{j}}=\frac{\lambda_{0}+\lambda_{1} \cos \theta^{j}}{\sin \theta^{j}} \frac{c^{j+1}-c^{j}}{c^{j} c^{j+1}}
$$

Therefore, since $\sin \theta^{j}=0$ implies $\lambda_{1}=-\lambda_{0}$ (Item 3 of Proposition 3.2), if $\sin \theta^{j}=0$ then $\lambda_{2}^{j+1} / c^{j+1}=$ $\lambda_{2}^{j} / c^{j}$. Furthermore,

$$
\left|\lambda_{3}^{j}(s)\right| c(s) \equiv \lambda_{2}^{j}(s) \sin (\theta(s))
$$

when $\sin \theta$ tends to 0 , this implies that $\lambda_{2}^{j}$ is of the same sign as $\sin (\theta(s))$ for $s$ around $s_{j+1}$. Hence $\lambda_{2}^{j+1} \leq \lambda_{2}^{j}$ for all $\theta^{j}$.

Two cases are now analyzed:

- Assume that the optimal path $\xi$ contains a line segment with orientation $\theta=\alpha \in[0, \pi]$. Then, $\lambda_{2}=$ $-\lambda_{0} \sin (\alpha) \leq 0\left(\lambda_{0}>0\right)$ along this line segment. Since $\lambda_{2}$ is decreasing along the whole path, there cannot exist any curvilinear abscissae along this path such that $\lambda_{2}=\lambda_{0} \sin (\alpha) \geq 0$. Therefore, the path cannot contain any reflections. Moreover, if the path contains another line segment with the same orientation $\alpha$, this means that $\lambda_{2}$ remains constant between these two segments. Thus, from Dubins' results [4], [1], the path between the two line segments is a whole circle. That kind of path is not optimal. Therefore, the optimal path $\xi$ contains only one line segment.

- Assume that the optimal path $\xi$ contains a line segment with orientation $\theta=\alpha \in[-\pi, 0]$. Then, $\lambda_{2}=$ $-\lambda_{0} \sin (\alpha) \geq 0\left(\lambda_{0}>0\right)$ along this line segment. Since $\lambda_{2}$ is decreasing along the whole path, there can exist a curvilinear abscissa along this path such that $\lambda_{2}=\lambda_{0} \sin (\alpha) \leq 0$. Therefore, the path can contain a reflection. Using the same previous argument from Dubins' results, the optimal path $\boldsymbol{\xi}$ can contain at most two line segments with both orientations $\alpha$ and $-\alpha$. 


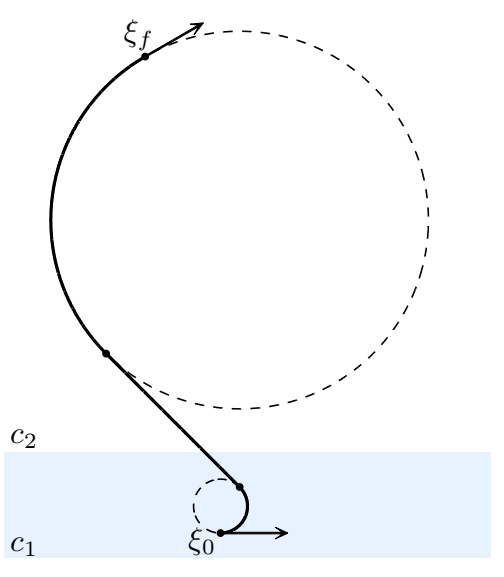

Fig. 2. Example 1 - CSC path with $\theta_{0}=0$ and $\theta_{f}=\frac{\pi}{6}$

Notice that the two line segments of an optimal path $\xi$ containing a reflection are necessarily joined by a unique arc $C$. This means that there is no inflection point between the line segments. This is due to the fact that $\lambda_{3}$ cannot vanish between the two line segments. The proof is straightforward using Item 3 of Proposition 3.2 and the fact that $\lambda_{2}$ is a decreasing function but the details are a little tedious to be presented here.

Furthermore, using symmetry arguments for the reflection, it can easily be shown that the derivative of the third adjoint variable $\lambda_{3}$ necessarily vanishes when $\sin \theta=0$ along the arc $C$ joining the two line segments. Thus, using Item 2 of Proposition 3.2, $\lambda_{2}$ also vanishes when $\sin \theta=0$ along this arc.

If $c(z)$ is a monotonic function, Theorem 3.4 implies that optimal paths are of type $C^{n_{1}} S_{\alpha} C^{n_{2}}, C^{n_{1}} S_{-\alpha} C S_{\alpha} C^{n_{2}}$ or a degenerated form of these, where $C^{n}(n \in \mathbb{N})$ denotes a concatenation of $n$ arcs of maximum curvature joined at inflection points $\left(\lambda_{3}=0\right)$ and $S_{\alpha}$ denotes a line segment with orientation $\alpha \in[-\pi, \pi]$. Maximum values for $n_{1}$ and $n_{2}$ are not obvious to determine a priori. Therefore, a geometric analysis need to be conducted on a case by case basis. Based on results for the Dubins' vehicle, the authors believe that $n_{1}=1$ and $n_{2}=1$, otherwise the path is not optimal. However, this remains an open problem.

Besides, analogously to Dubins' results, there can exist optimal paths without any line segments, i.e. there can exist optimal paths of type $C^{n}$. For Dubins' paths, it was shown that $n$ cannot be superior to 3 . In the case where $c(z)$ is a monotonic function, this remains an open question.

In next two sections, some optimal paths of type $C S_{-\alpha} C S_{\alpha} C$ and $C S_{\alpha} C$ are presented for two concrete application cases.

\section{EXAMPLE 1: A DISCONTINUOUS ENVIRONMENT}

In this section, the vehicle considered is a mobile robot which dynamics verify (1) with $c(z)=\tan \delta_{\max } / L$, where $\delta_{\max }$ is the maximum value of the steering angle and $L$ is the distance between front and rear wheels. The environment consists of two half-planes where the coefficient of friction

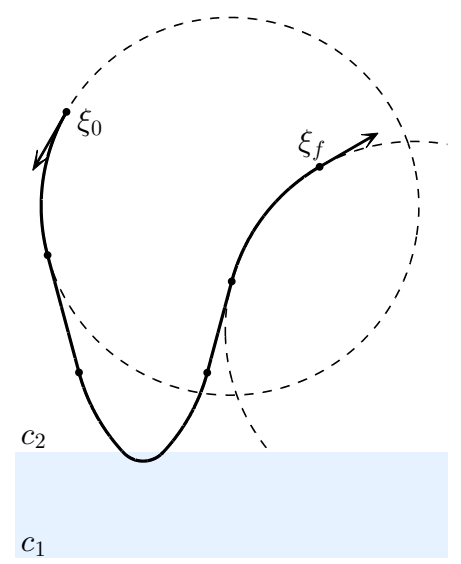

(a) $\theta_{0}=-\frac{2 \pi}{3}$ and $\theta_{f}=\frac{\pi}{6}$

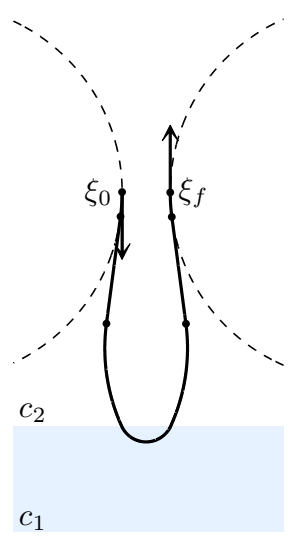

(b) $\theta_{0}=-\frac{\pi}{2}$ and $\theta_{f}=$
Fig. 3. Example 1 - CSCSC paths

between the robot and the ground is different. As the vehicle moves at a constant speed and is assumed to roll without skidding nor sliding, it implies that the maximum steering angle that the robot can perform differs between the two half-planes. Therefore, the maximum curvature $c$ is different for the two half-planes.

The glider presented in next section $\mathrm{V}$ verifies the same kind of model if densities of the fluid in both half planes are different. For example, if the first half plane consists of water and the second half plane consists of air, the density is different for the two half-planes then the maximum curvature $c$ is different for the two half-planes.

In the followings, the maximum curvature $c_{1}$ of the first halfplane will be considered superior to the maximum curvature $c_{2}$ of the second half-plane.

Results of Section III can be used. In particular, Theorem 3.4 implies that optimal paths with line segments can contain reflections, i.e. optimal paths can contain two line segments with opposite orientation angles. To perform a reflection, the robot needs to cross the discontinuity two times with opposite orientation angles. Using Equation (3) for both switching curvilinear abscissae, it can be shown that the absolute value $\left|\theta_{s}\right|$ of switching orientation angles verifies

$$
\sin \left|\theta_{s}\right| \sin |\alpha|=\left(1-\cos \left|\theta_{s}\right| \cos |\alpha|\right) \frac{c_{2}-c_{1}}{c_{1}}
$$

recalling that $|\alpha|$ is the absolute value of the orientation angle of both line segments. Notice that a reflection can be optimal only if both line segments lie in the half-plane with the greater curvature, i.e. the half-plane with curvature $c_{2}$ in this example (see proof of Theorem 3.4 for more details).

Considering Bellman's principle of optimality, notice that Dubins' results must be verified in each half-plane, i.e. any pieces of an optimal path lying in a single half-plane is necessarily of type $C C C$ or $C S C$.

Figures 2 and 3 present some optimal paths with line segments choosing $c_{1}=7 c_{2}$. The first half-plane with maximum curvature $c_{1}$ is represented in blue on these figures. Moreover, any changes of control input $u \in\{-1,0,1\}$ is 
marked by a black dot along the path. Figure 2 presents a $C S C$ optimal path starting from the first half-plane with initial orientation angle $\theta_{0}=0$ and ending in the second half-plane with final orientation angle $\theta_{f}=\pi / 6$. Figure 3 presents two CSCSC optimal paths starting and ending in the second half-plane. For these two paths, notice that if the environment were homogeneous with maximum curvature $c_{2}$, the optimal path would be of type $C C C$ due to the fact that initial and final positions are close to each other compared to the minimum turning radius. However, taking advantage of the greater curvature in the first half-plane, $C S C S C$ paths are shorter here.

\section{EXAMPLE 2: A CONTINUOUS ENVIRONMENT}

In this section, the vehicle considered is a glider of mass $m$ flying in the $2 \mathrm{D}$ vertical plane. The vehicle forward velocity $v$ is assumed to be high enough in order to neglect the gravitational force during the flight. Typically, a missile can be assimilated to an hypersonic glider after its propulsion stage. Thus, only the force of lift $f_{L}$ contributes to maneuver the aerial vehicle. This can be written as follows:

$$
f_{L}=\frac{1}{2} \rho(z) S C_{L} v^{2}
$$

where $\rho(z)$ is the air density depending on the current altitude, $S$ is the reference area of the glider and $C_{L}$ is the lift coefficient depending on the angle of attack. Using the fact that the rotational velocity verifies $\dot{\theta}=f_{L} / m v$, the dynamics of the glider satisfy equations (1) with $c(z)=$ $\frac{1}{2 m} \rho(z) S\left(C_{L}\right)_{\max }$. The environment is heterogeneous since $\rho(z)$ depends on the altitude. In the followings, it is modeled as an exponentially decreasing function of altitude: $\rho(z)=$ $\rho_{0} \exp \left(-\frac{z}{z_{r}}\right)$ with $z_{r}=7500 \mathrm{~m}$.

To derive arcs of maximum curvature, the control input $u$ is chosen such that $|u|=1$. Differentiating $\theta^{\prime}$ with respect to $s$, this yields:

$$
\theta^{\prime \prime}=-\frac{1}{z_{r}} \theta^{\prime} \sin \theta
$$

Thus, closed-form solutions for $\theta, x$ and $z$ can be obtained. Let define $\zeta=\tan \left(\frac{\theta}{2}\right)$ and differentiate it. After some straightforward but tedious calculations, one obtains

$$
\begin{aligned}
\zeta^{\prime} & =A+B \zeta^{2}, \\
A & =\frac{1}{2 z_{r}}\left(z_{r} \theta_{0}^{\prime}-\cos \theta_{0}+1\right), \\
B & =A-\frac{1}{z_{r}} .
\end{aligned}
$$

Therefore, four types of curves can be deduced depending on values of $A$ and $B$ :

1) $C_{1}$ type of curve if $A B>0$,

$$
\zeta_{1}(s)=\sqrt{\frac{A}{B}} \tan \left[A \sqrt{\frac{B}{A}} s+\arctan \left(\sqrt{\frac{B}{A}} \zeta_{0}\right)\right]
$$

This type of curve is illustrated in Figure 4.

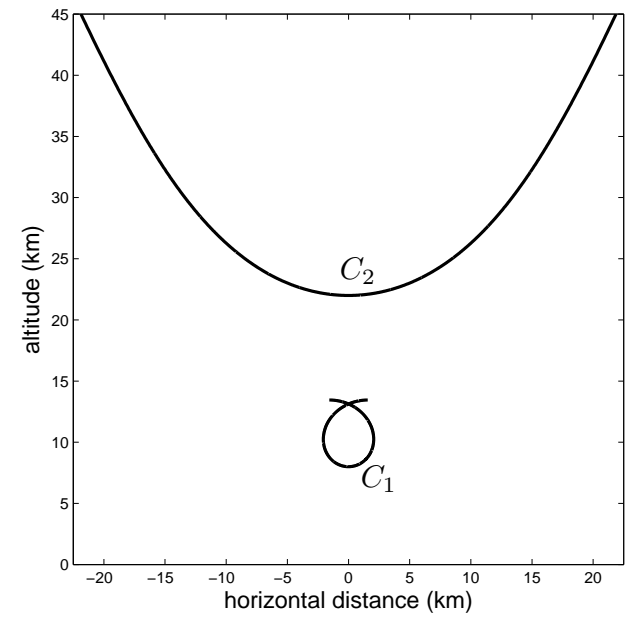

Fig. 4. Example 2 - Arcs of maximum curvature

2) $C_{2}$ type of curve if $A B<0$,

$\zeta_{2}(s)=\sqrt{\left|\frac{A}{B}\right|} \tanh \left[A \sqrt{\left|\frac{B}{A}\right|} s+\operatorname{arctanh}\left(\sqrt{\left|\frac{B}{A}\right|} \zeta_{0}\right)\right]$

This type of curve is illustrated in Figure 4. Notice that this type of curve has asymptotes.

3) $C_{3}$ type of curve if $A=0$,

$$
\frac{1}{\zeta_{3}(s)}=\frac{1}{\zeta_{0}}-B s
$$

4) $C_{4}$ type of curve if $B=0$,

$$
\zeta_{4}(s)=\zeta_{0}+A s
$$

$\theta, x$ and $z$ can be deduced from the expression of $\zeta$ as follows:

$$
\left\{\begin{array}{l}
\theta(\zeta)=2 \arctan \zeta \\
x(\zeta)=x_{0}+z_{r}\left(\theta(\zeta)-\theta_{0}\right)-z_{r}(A+B) s(\zeta) \\
z(\zeta)=z_{0}-z_{r} \ln \left(\frac{1+\zeta_{0}^{2}}{A+B \zeta_{0}^{2}} \frac{A+B \zeta^{2}}{1+\zeta^{2}}\right)
\end{array}\right.
$$

Once again, Theorem 3.4 applies. Therefore, optimal paths can be of type $C S C$ and $C S C S C, C$ denoting one of the four types of arcs presented above. For this application case, a reflection can be renamed a rebound.

Figures 5, 6 and 7 present some paths with line segments that are candidates for optimality. The maximum curvature at altitude zero is chosen as $c(0)=0.0015 \mathrm{~m}^{-1}$, it depends on the glider parameters. Figure 5 presents a $C_{1} S C_{1}$ path starting close to the ground with initial orientation angle $\theta_{0}=$ 0 and ending at an altitude greater than $20 \mathrm{~km}$ with final orientation angle $\theta_{f}=\pi / 12$. Figure 6 presents a $C_{2} S C_{2}$ path starting and ending at high altitude with initial orientation angle $\theta_{0}=-\pi / 3$ and final orientation angle $\theta_{f}=\pi / 3$. 


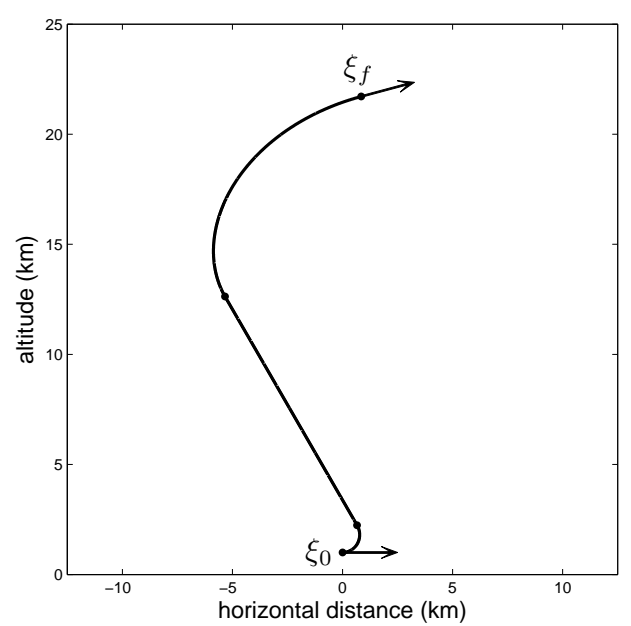

Fig. 5. Example 2 - CSC path with $\theta_{0}=0$ and $\theta_{f}=\frac{\pi}{12}$

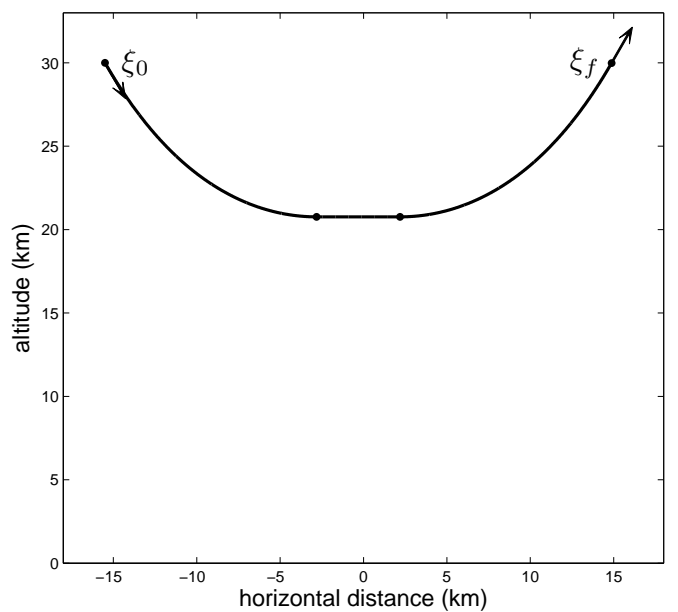

Fig. 6. Example 2 - CSC path with $\theta_{0}=-\frac{\pi}{3}$ and $\theta_{f}=\frac{\pi}{3}$

Figure 7 presents two $C S C S C$ paths starting and ending at high altitude.

\section{CONCLUDING REMARKS}

This paper presented the problem of minimizing path length in an heterogeneous environment for a Dubins' vehicle moving forward and steering with a maximum curvature that depends on the vehicle position. Some necessary conditions for optimality were deduced applying optimal control theory. These provide practical conditions to build optimal paths for such a system as well as an interesting result that generalizes reflection law to the system considered. Two examples of application are described to illustrate the approach and to demonstrate its usefulness for practical implementations such as path planning in complex environments [7].

\section{REFERENCES}

[1] J.-D. Boissonnat, A. Cérézo, and J. Leblond. Shortest paths of bounded curvature in the plane. Technical report, RR-1503 INRIA, 1991.

[2] J.-D. Boissonnat, A. Cérézo, and J. Leblond. A note on shortest paths in the plane subject to a constraint on the derivative of the curvature. Technical report, RR-2160 INRIA, 1994
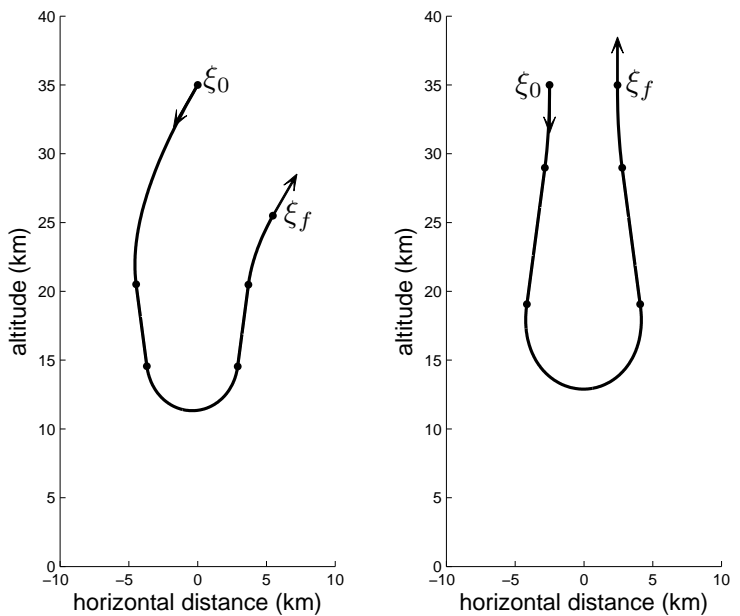

(a) $\theta_{0}=-\frac{2 \pi}{3}$ and $\theta_{f}=\frac{\pi}{3}$

(b) $\theta_{0}=-\frac{\pi}{2}$ and $\theta_{f}=\frac{\pi}{2}$

Fig. 7. Example 2 - CSCSC paths

[3] I. Dolinskaya and A. Maggiar. Time-optimal trajectories with bounded curvature in anisotropic media. The International Journal of Robotics Research, 2012.

[4] L. E. Dubins. On curves of minimal length with a constraint on average curvature and with presribed initial and terminal position and tangents. American Journal of Mathematics, 79:497-516, 1957.

[5] T. G. McGee, J. K. Hedrick, S. M. LaValle, F. Bullo, M. W. Spong, J. P. How, and E. W. Frew. Optimal path planning with a kinematic airplane model. Journal of guidance, control, and dynamics, 30(2):629-633, 2007.

[6] T. G. McGee, S. Spry, and J. K. Hedrick. Optimal path planning in a constant wind with a bounded turning rate. In Proceedings of the AIAA Guidance, Navigation, and Control Conference and Exhibit, pages $1-11,2005$.

[7] P. Pharpatara, R. Pepy, B. Hérissé, and Y. Bestaoui. Missile trajectory shaping using sampling-based path planning. In Proceedings of the IEEE/RSJ International Conference on Intelligent Robots and Systems, Tokyo, Japan, 2013. To appear.

[8] L. S. Pontryagin, V. G. Boltyanskii, R. V. Gamkrelidze, and E. F. Mishchenko. The mathematical theory of optimal processes. Wiley, New York, 1962.

[9] J. A. Reeds and L. A. Shepp. Optimal paths for a car that goes both forwards and backwards. Pacific Journal of Mathematics, 145:367393, 1990.

[10] R. Sanfelice and E. Frazzoli. On the optimality of dubins paths across heterogeneous terrain. Hybrid Systems: Computation and Control, pages 457-470, 2008.

[11] H. J. Sussmann. Shortest 3-dimensional paths with a prescribed curvature bound. In Proceedings of the IEEE Conference on Decision and Control, pages 3306-3312, 1995.

[12] H. J. Sussmann. A maximum principle for hybrid optimal control problems. In Proceedings of the IEEE Conference on Decision and Control, pages 425-430, 1999.

[13] H. J. Sussmann and G. Tang. Shortest paths for the reeds-shepp car: a worked out example of the use of geometric techniques in nonlinear optimal control. Technical report, Rutgers Center for Systems and Control, 1991. 